\title{
Effect of exercise on erythrocyte osmotic fragility in high fat diet fed rats during intermittent fasting
}

\author{
Semih Yaşar ${ }^{1}$, Nizamettin Günbatar ${ }^{1}$, Sevgi Yüksek ${ }^{2}$, Okan Arıhan $^{3^{*}}$, Gökhan Oto $^{2}$ \\ ${ }^{1} V$ an Yuzuncu Yil University, Faculty of $V$ an Vocational School of Health, Van, Turkey \\ ${ }^{2} V$ an Yuzuncu Yil University, Faculty of Medicine, Department of Pharmacology, Van, Turkey \\ ${ }^{3} V$ an Yuzuncu Yil University, Faculty of Medicine, Department of Physiology, Van, Turkey
}

\begin{abstract}
Exercise is an important component of a healthy lifestyle. High fat diets are blamed for various health problems such as cardiovascular diseases. Intermittent fasting is a way of increasing longevity and decreasing certain adverse health conditions. In this study effect of exercise, intermittent fasting and high fat diet on erythrocyte osmotic fragility which is an important marker of erythrocyte integrity was investigated.

42 Wistar albino male rats were used in this study. Rats were divided into 7 groups in which each were composed of 6 animals. Exercise was administered 3 times a week. Intermittent fasting was administered 2 times a week (24 without any food in those two days). High fat was given in a high fat diet. Combination of applications was also administered in different groups.

Results state an increase in erythrocyte osmotic fragility due to exercise. High fat or intermittent fasting did not cause in increase in fragility however they did not alleviate increased fragility due to exercise where they are concomitantly administered with exercise. Intermittent fasting administered solely or with high fat did not change erythrocyte osmotic fragility therefore stating no negative impact of intermittent fasting on this studied parameter. However no protective effect was observed in IF+Exe group. This result can also be interpreted with high impact of Exe in this study on the rats. Studies including molecular mechanism focusing on erythrocyte membrane lipid alterations should be performed to clarify current findings.
\end{abstract}

Key Words: Intermittent fasting, exercise, high fat diet, erythrocyte fragility

\section{Introduction}

High fat including diets are deleterious for general health and deteriorate health body composition. A healthy diet and exercise is advised for maintaining a healthy life. Exercise is protective against many diseases and prevents obesity, osteoporosis and cardiovascular diseases such as hypertension (1). Regular moderate exercise is good for various aspects of health such as mood alleviation (2). However, irregular and exhausting exercises are not protective and even destructive for cardiovascular system and immune system as well as causing musculoskeletal injuries (3).

During decades efforts to slowdown aging which are grouped under anti-aging supports intermittent fasting as one of the most scientifically proven and efficient way. Even in short time caloric restriction is shown to be protective against cardiovascular diseases and cancer (4-7). Since there exists a risk of malnutrition in prolonged fasting schemes intermittent fasting seems like an applicable solution which is also supported by data from scientific studies on humans and animal models (8-12). Intermittent fasting is also shown to exert protective effect against cancer $(13,14)$.
This study was performed to evaluate effect of exercise, high fat diet and intermittent fasting and their concomitant administration on erythrocyte osmotic fragility test which is a functional assessment test for erythrocyte integrity and condition.

\section{Materials and methods}

Experimental design: 42 Wistar albino male rats were used in this study. Rats were divided into 7 groups in which each were composed of 6 animals. Control group was fed with standard rat pellet ad libitum. Exercise group (Exe) was administered exercise (3 times a week). In intermittent fasting group (If) animals were fasted for 2 times a week (24 without any food in those two days). Animals in high fat group (Hfat) received a diet composing high fat (with $60 \%$ of energy coming from prepared pellet including hydrogenated saturated fats purchased from commercial market) daily for 6 weeks. $5^{\text {th }}$ group received exercise and high fat $\operatorname{diet}(\mathrm{Hfat}+\mathrm{Exe})$. $6^{\text {th }}$ group was administered with high fat diet and intermittent fasting (Hfat+IF). $7^{\text {th }}$ group was administered with intermittent fasting and exercise (IF+Exe). 
Erythrocyte osmotic fragility test: Blood samples were obtained into EDTA containing tubes at the end of $8^{\text {th }}$ week. Erythrocyte osmotic fragility was measured with a spectrophotometric device (Prime$\mathrm{Ev}, \mathrm{BPC})$. Method is modified from Beutler (15). Dilutions of $0.1,0.4,0.5$ and $0.9 \% \mathrm{NaCl}$ were prepared ( $\mathrm{pH}$ 7.4). $30 \mu \mathrm{L}$ of blood was transferred into dilution tubes containing $2 \mathrm{~mL}$ of the prepared solutions. Blood was then mixed with the solutions. Following $30 \mathrm{~min}$ of incubation at an ambient temperature, the tubes were centrifuged for $5 \mathrm{~min}$ $(3000 \mathrm{rpm})$. Supernatants were placed into the spectrophotometer and measured at $546 \mathrm{~nm}$.

Exercise tests: Tests were performed on rat treadmill. For an adaptation period, rats were allowed to run on the lowest speed for 2 weeks. Following adaptation 8 weeks of treadmill exercise was administered for the exercise groups 3 times per week according to Rico et al (16). Exercise was administered as moderate intensity (30 minutes) with as speed of $40 \mathrm{~cm} / \mathrm{s}$.

Statistical Analysis: Results are expressed as mean of percent hemolysis. For statistical analysis SPSS (IBM) software was used. Oneway Anova and Duncan tests were applied. Statistical significance was set to $\mathrm{p}<0.05$.

\section{Results}

Results show an increase of erythrocyte osmotic fragility compared to control due to exercise administration. In $0.4 \% \mathrm{NaCl}$ all of the exercise groups had highest fragility values among all tested groups. Exe, Hfat+Exe and IF+Exe groups were found significantly higher compared to control $(p<0.05)$. IF, Hfat and Hfat+If groups were found similar to control group ( $p>0.05)$. In $0.5 \% \mathrm{NaCl}$ again Exe, Hfat+Exe and IF+Exe groups were found significantly higher compared to control $(\mathrm{p}<0.05)$. In this concentration Hfat, IF and control groups were again found similar $(\mathrm{p}>0.05)$ (Table 1$)$.

Table 1. Erythrocyte osmotic fragility values of groups

\begin{tabular}{lcccc}
\hline & 0.1 & 0.4 & 0.5 & 0.9 \\
Control & 100 & $56^{\mathrm{b}}$ & $46^{\mathrm{c}}$ & 0 \\
Exe & 100 & $83^{\mathrm{a}}$ & $62^{\mathrm{b}}$ & 0 \\
IF & 100 & $65^{\mathrm{ab}}$ & $48^{\mathrm{bc}}$ & 0 \\
Hfat & 100 & $67^{\mathrm{ab}}$ & $42^{\mathrm{c}}$ & 0 \\
Hfat+Exe & 100 & $87^{\mathrm{a}}$ & $77^{\mathrm{a}}$ & 0 \\
Hfat+IF & 100 & $73^{\mathrm{ab}}$ & $61^{\mathrm{b}}$ & 0 \\
IF+Exe & 100 & $79^{\mathrm{a}}$ & $63^{\mathrm{b}}$ & 0 \\
\hline
\end{tabular}

Results are presented as percent hemolysis as mean. Exe: exercise group, IF: intermittent fasting group, Hfat: high fat fed group, Hfat+Exe: high fat fed+ exercise group, Hfat + If: high fat fed + intermittent fasting group, IF+Exe: intermittent fasting + exercise group. Data was statistically evaluated by oneway Anova and Duncan tests. Significance was set to $\mathrm{P}<0.05$. Values of groups with different lowercase letters $(a, b, c)$ in the same column and line are significant.

\section{Discussion}

Increased physical activity and in the form of planned exercise schedules is shown to support general health and advised by health care professionals to every age and sex (17). Although exercise is increasing antioxidant status, positive for mood, protects cardiovascular health and good for alleviating altered lipid profile it may pose some threats to health (18). When conducted without precaution it may cause cardiovascular and musculoskeletal problems (19). Results of this study show an increase in erythrocyte osmotic fragility due to exercise in this experimental setup. Compared to control group all exercise administered groups had higher erythrocyte osmotic fragility in $0.4 \% \mathrm{NaCl}$ concentration. On the other hand intermittent fasting or high fat diets had no significant impact compared to control. Similarly in In $0.5 \% \mathrm{NaCl}$ Exe, Hfat+Exe and IF+Exe groups were found significantly higher compared to control $(p<0.05)$. Results of this study show that exercise in this experimental design increased erythrocyte osmotic fragility of rats. As observed in human subjects exercise poses increased oxidative stress also in rats during adaptation period to exercise. In addition during this adaptation interval, erythrocyte osmotic fragility was also found higher in other studies (20). However after such an adaptation period, exercise was found to exert no significant increase on erythrocyte osmotic fragility (20). A similar situation was also observed in humans which shows exercise is beneficial for trained individuals but exerting untoward effect on sedentary ones (21). Our findings can be interpreted as insufficient adaptation of rats to exercise although a preparatory exercise period was administered prior to exercise.

Lipids are important components of erythrocyte membrane. Any deficiency of lipids and essential oils were shown to increase erythrocyte osmotic fragility (22). On the other hand cholesterol which is also commonly and concurrently ingested with lipids was also shown to deteriorate integrity of erythrocytes in osmotic fragility test (23). High fat intake in dogs was also shown to increase erythrocyte fragility (24). On 
the other hand there are studies in which high fat diets was found to protect erythrocytes slightly against osmotic lysis of the membrane by increasing stability of erythrocyte membrane (25). In this study no such protection was observed in Hfat+Exe group compared to Exe group. This can be due to high impact of Exe in this study on the rats or difference in administered fat type. In literature fats from animal origin was used for high fat diet whereas in this study hardened margarine was used which can be the reason of dissimilar result with literature.

In conclusion; intermittent fasting administered solely or with high fat did not change erythrocyte osmotic fragility therefore stating no negative impact of intermittent fasting on this studied parameter. However no protective effect was observed in IF+Exe group. This result can also be interpreted with high impact of Exe in this study on the rats. Studies including molecular mechanism focusing on erythrocyte membrane lipid alterations should be performed to clarify current findings.

\section{References}

1. Levine GN, Balady GJ. The benefits and risks of exercise training: The exercise prescription. In Levy WC, Cerqueira MD, Abrass IB, Schwartx RS, Stratton JR Eds Advances in Internal Medicine Springer 38: Pp 57-79.

2. Harvey SB, Øverland S, Hatch SL, Wessely S, Mykletun A, Hotopf M. Exercise and the Prevention of Depression: Results of the HUNT Cohort Study. Am J Psychiatry 2017 Oct 3:appiajp201716111223. doi: 10.1176/appi.ajp.2017.16111223.

3. Pyne DB, Gleeson M. Effects of intensive exercise training on immunity in athletes. Int J Sports Med 1998; 19: 183-194.

4. Walford RL, Mock D, MacCallum T, Laseter JL. Physiologic changes in humans subjected to severe, selective calorie restriction for two years in biosphere 2: health, aging, and toxicological perspectives. Toxicol Sci 1999; 52: 61-65.

5. Weyer C, Walford RL, Harper IT, et al. Energy metabolism after 2 y of energy restriction: the biosphere 2 experiment. Am J Clin Nutr 2000; 72: 946-953.

6. Michels KB, Ekbom A. Caloric restriction and incidence of breast cancer. JAMA 2004; 291: 12261230.

7. Elias SG, Peeters PH, Grobbee DE, van Noord PA. Transient caloric restriction and cancer risk (the Netherlands). Cancer Caus Cont 2007; 18: 1-5.

8. Sogawa H, Kubo C. Influence of short-term repeated fasting on the longevity of female (NZB x NZW) F1 mice. Mech Ageing Dev 2000; 115: 61-71.

9. Berrigan D, Perkins SN, Haines DC, Hursting SD. Adult-onset calorie restriction and fasting delay spontaneous tumorigenesis in p53-deficient mice. Carcinogenesis 2002; 23: 817-822.

10. Cleary MP, Jacobson MK, Phillips FC, Getzin SC, Grande JP, Maihle NJ. Weight-cycling decreases incidence and increases latency of mammary tumors to a greater extent than does chronic caloric restriction in mouse mammary tumor virus transforming growth factor-alpha female mice. Cancer Epidemiol Biomark Prev 2002; 11: 836-843.

11. Anson RM, Guo Z, de Cabo R, A et al. Intermittent fasting dissociates beneficial effects of dietary restriction on glucose metabolism and neuronal resistance to injury from calorie intake. Proc Natl Acad Sci USA 2003; 100: 6216-6220.

12. Bonorden MJ, Rogozina OP, Kluczny CM, et al. Cross-sectional analysis of intermittent versus chronic caloric restriction in the TRAMP mouse. Prostate 2009; 69: 317-326.

13. Hsieh EA, Chai CM, Hellerstein MK. Effects of caloric restriction on cell proliferation in several tissues in mice: role of intermittent feeding. Am J Physiol Endocrinol Metab 2005; 288: 965-972.

14. Varady KA, Roohk D, Hellerstein MK. Dose effects of modified alternate-day fasting regimens on in vivo cell proliferation and plasma insulin-like growth factor-1 in mice. J Appl Physiol 2007; 103: 547-551.

15. Beutler E. Osmotic fragility. In: Hematology, edited by Williams. WJ, Beutler E, Erslev AJ, and Lichtman MA. New York: McGraw-Hill, 1983; 1626-1627.

16. Rico H, Gervas J, Hernandez ER, Seco C, Villa LF, Revilla M. Effect of alprazolam supplementation on vertebral and femoral bone mass in rats on strenuous treadmill training exercise Calcif Tissue Int 1999 65; 139-142.

17. Doughty KN, Del Pilar NX, Audette A, Katz DL. Lifestyle Medicine and the Management of Cardiovascular Disease. Curr Cardiol Rep 2017 4; 19: 116.

18. Gleason PT, Kim JH. Exercise and Competitive Sport: Physiology, Adaptations, and Uncertain LongTerm Risks. Curr Treat Options Cardiovasc Med 2017; 19: 79.

19. Green B, Pizzari T. Calf muscle strain injuries in sport: a systematic review of risk factors for injury. $\mathrm{Br}$ J Sports Med 2017; 51:1189-1194.

20. Senturk UK, Gunduz F, Kuru O, et al. Exerciseinduced oxidative stress affects erythrocytes in sedentary rats but not exercise-trained rats. J Appl Physiol 2001; 91: 1999-2004.

21. Senturk UK, Gunduz F, Kuru O, et al. Exerciseinduced oxidative stress leads hemolysis in sedentary but not trained humans. J Appl Physiol 2005; 99: 1434-1441.

22. Ehrstrom M, Harms-Ringdahl M, Alling C. Osmotic fragility and fluidity of erythrocyte membranes from rats raised on an essential fatty acid deficient diet. Biochimica et biophys 1981; 644: 175-182. 
Yaşar et al / Erythrocyte fragility in exercise, fasting and high fat diet

23. Kempaiah RK, Srinivasan K. Integrity of erythrocytes of hypercholesterolemic rats during spices treatment. Mol Cel Biochem 2002; 236: 155-161.

24. Longini J, Johnson V. Increased red blood cell fragility alter fat ingestion. Am J Physiol 1943; 140: 349-363.
25. Kempaiah RK, SrinivasanK. Influence of Dietary Curcumin, Capsaicin and Garlic on the Antioxidant Status of Red Blood Cells and the Liver in High-FatFed Rats. Ann Nutr Metab 2004; 48: 314-320. 